\title{
Estudio socioeducativo de los jóvenes internados en las prisiones andaluzas.
}

\author{
Víctor Manuel Martín Solbes \\ Universidad de Málaga \\ victorsolbes@uma.es
}

\section{RESUMEN}

Con este artículo sintetizamos una investigación con la que intentamos conocer el perfil socioeducativo de los jóvenes que ocupan las prisiones andaluzas. Creemos que conociendo este perfil y actuando desde la educación y la prevención podemos evitar futuros ingresos en prisión. Para realizar el estudio analizamos una serie de variables socioeducativas que creemos importantes; asimismo, utilizamos una escala de sinceridad que nos ayude a identificar y eliminar a los sujetos menos sinceros. Tras estos análisis observamos, entre otras conclusiones, el bajo nivel académico, el abandono escolar prematuro, el alto porcentaje de consumidores de drogas, así como la escasa o nula cualificación profesional de la población estudiada.

\begin{abstract}
With this article we synthesized an investigation with which we tried to know the profile socioeducative about the young people who live the andalusian prisons. We believe that if we know his profile and acting from the education and the prevention, we can avoid futures income in prison. In order to make the study we analyzed a series of socioeducatives variables that we think they are important; also, we used a sincerity scale that helps us to identify and to eliminate the less sincere subjects. After these analyses we observed, among other conclusions, the low academic level, premature the scholastic abandonment, the high percentage of drug users, as well as the little or null professional qualification of the studied population.
\end{abstract}




\section{Introducción}

Con este artículo tratamos de sintetizar una investigación con la que pretendíamos conocer el perfil socioeducativo de los jóvenes menores de veintiún años internados en los centros penitenciarios andaluces.

Nos hemos centrado en los jóvenes menores de veintiún años, basándonos en cinco criterios:

a.- Penitenciarios, ya que deseamos estudiar a los jóvenes en prisión, y el artículo 173 del vigente Reglamento Penitenciario Español, aprobado por Real Decreto 190/1.996 de 9 de febrero, expresa en su punto 1:

"El régimen de vida de los departamentos para jóvenes se caracterizará por una acción educativa intensa. Se consideran jóvenes a los internos menores de veintiún años”.

b.- Criminológicos, porque la etapa que va desde la infancia o preadolescencia hasta los veintiún años, corresponde a la franja más activa en la carrera delictiva de un sujeto. Es en esta etapa donde se producen más conductas antisociales, se cometen más delitos, donde el grado de reincidencia es mayor y donde se refleja un riesgo más elevado en lo que se refiere a la peligrosidad social de sus actos (Kaiser, 1.988).

c.- Madurativos, porque al tratarse de delincuentes jóvenes, ellos son los que van a nutrir el sistema penitenciario en los próximos años, si siguen teniendo pautas delincuenciales (Beristain, 1.985).

d.- Pedagógicos, ya que se trata de una etapa en la que la educación puede realizar una labor reeducadora fundamental, evitando así futuros ingresos en prisión (Martín, 2006).

e.- Psicosociales, porque a estas edades es posible la inserción, tanto social como laboral, siendo ésta una etapa de construcción de la identidad en el contexto social (García y Sancha, 1.986).

En el desarrollo del estudio hemos tenido muy presente que "la penas privativas de libertad estarán orientadas hacia la reeducación y la reinserción social" (artículo 25.2 de la Constitución Española) y las posibilidades que la Institución Penitenciaria ofrece a los jóvenes internados para que este precepto tenga una consecución real. 


\section{Método}

Para llevar a cabo la investigación nos hemos centrado en el estudio de los internos jóvenes de los centros penitenciarios de Sevilla, Algeciras, Málaga y Granada. Y lo hemos hecho así porque en el momento de realizar la investigación, la población internada menor de veintiún años en los centros penitenciarios andaluces, era de 334 internos, de los que 311 habitaban en los centros penitenciarios antes citados. Aun siendo conscientes de la posibilidad de que características individuales han podido quedar fuera del estudio, proponemos a estos 311 jóvenes participar en la investigación, aceptando la propuesta 285 internos que se distribuían de la manera que se expresa en la tabla 1.

Tabla 1.- Centros penitenciarios visitados, número y porcentajes de internos

\begin{tabular}{|c|c|c|c|c|c|c|}
\hline & N español & $\%$ & N extranj. & $\%$ & N total & $\%$ \\
\hline SEVILLA & 64 & 22,4 & 19 & 6,7 & 83 & 29,1 \\
\hline ALGECIRAS & 49 & 17,3 & 27 & 9,4 & 76 & 26,7 \\
\hline MALAGA & 42 & 14,6 & 23 & 8,2 & 65 & 22,8 \\
\hline GRANADA & 46 & 16,1 & 15 & 5,3 & 61 & 21,4 \\
\hline Total español & $\mathbf{2 0 1}$ & $\mathbf{7 0 , 4}$ & & & & \\
\hline Total extranj. & & & $\mathbf{8 4}$ & $\mathbf{2 9 , 6}$ & & \\
\hline TOTAL & & & & & $\mathbf{2 8 5}$ & $\mathbf{1 0 0}$ \\
\hline
\end{tabular}

Para llevar a cabo la investigación nos hemos valido de un cuestionario de variables socioeducativas y de una escala de sinceridad.

\subsection{Escala de sinceridad}

La exponemos en primer lugar porque esto facilita el posterior desarrollo de la exposición de la investigación.

Introducimos esta escala por necesidad metodológica, para identificar a los sujetos poco sinceros de nuestra investigación y proceder a su eliminación, evitando así extraer conclusiones erróneas. Utilizamos la subescala de sinceridad propuesta por Pinillos en el cuestionario CEP de personalidad (1.990), que está constituida por una 
serie de ítems referidos a cuestiones relativas a pequeñas faltas morales o sociales frecuentes, pero cuya confesión pública puede resultar incómoda.

Una vez recogidas las respuestas de los cuestionarios, abordamos el análisis de los mismos con el programa estadístico SPSS versión 11.5.

Con objeto de establecer un punto de corte que permita distinguir a los jóvenes que son más sinceros de los que lo son menos, se ha obtenido el percentil 25 , cuyo valor nos da una puntuación de 10; el percentil 50, cuyo valor es 13 y el percentil 75, cuyo valor es 16 .

Figura 1.- Distribución de puntuaciones en sinceridad

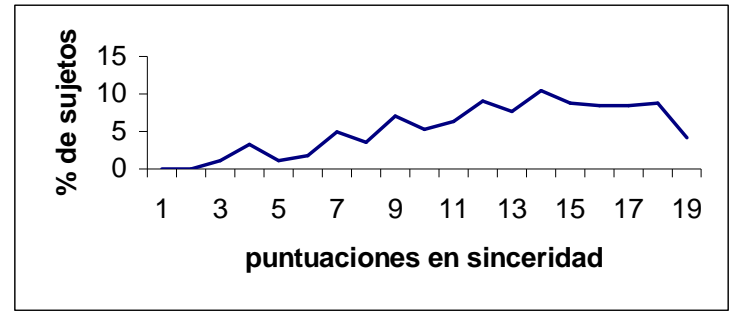

A partir de estos valores procedemos a eliminar los casos que se encuentran por debajo del percentil 25 , por considerar que estos jóvenes han sido poco sinceros en sus respuestas, quedando el número de casos reducido a 206 sujetos, que podemos considerar que han sido más sinceros en sus respuestas. Observamos que el 27,7\% de los jóvenes han sido poco sinceros.

De estos 206 sujetos, 196 son hombres y 10 son mujeres. Son españoles el $70,5 \%$, estableciéndose la edad media en 19 años.

\subsection{Variables socioeducativas}

Hemos seleccionado una serie de variables que consideramos que de alguna manera pueden indicarnos el perfil socioeducativo de los jóvenes que estudiamos y con ellas pretendemos conocer tanto las singularidades de los jóvenes como las características del grupo.

Estas variables socioeducativas las agrupamos en cuatro ámbitos de estudio: 
- Nivel académico y cualificación profesional, donde se recogen datos relativos a la edad, nivel académico, formación, experiencia laboral, etc.

- Relaciones familiares, donde se recoge información sobre los estudios de los padres y madres, las profesiones paternas y maternas, si existen o no antecedentes familiares en prisión, etc.

- Contactos con la marginalidad, recogiendo datos relativos a consumos de drogas, primeros consumos, tipos de drogas consumidas, primer delito cometido, delitos posteriores, influencias recibidas para delinquir, ingresos en centros de menores, etc.

- Vida en prisión, con la que pretendemos conocer el itinerario en el interior del centro penitenciario, recogiendo datos relativos a la situación penal, si están en situación de penados o de preventivos, a la espera de juicio; situación penitenciaria, si son primarios o reincidentes, con más de un ingreso en prisión; actividad que realiza en prisión, si estudia, trabaja, en qué ocupa el tiempo libre, etc.

En esta investigación nos planteamos estudiar a los jóvenes menores de veintiún años en prisión. Con la aparición de la Ley Orgánica 5/2.000, de 12 de enero, reguladora de la responsabilidad penal de los Menores, queda suprimida la anterior normativa que permitía que los jóvenes de 16 y 17 años pudieran entrar en prisión, por lo que el tramo de edad que estudiamos es el que abarca desde los 18 hasta los 21 años, es decir, internos de 18, 19 y 20 años.

Podemos apreciar que en la muestra con la que trabajamos coexisten internos españoles payos y de etnia gitana, extranjeros, ya sean europeos, del magreb, subsaharianos, asiáticos o de América, tanto latina como del norte. Asimismo, coexisten hombres y mujeres, primarios y reincidentes, cuya procedencia social es variada y aunque predominan los pertenecientes a las clases más desfavorecidas, cada vez es más importante el número de personas que pertenecen a clases normalizadas socialmente y que ingresan en prisión por unos u otros motivos.

La muestra con la que trabajamos representa el $85 \%$ de la población penitenciaria andaluza a la que va dirigida el estudio.

A continuación describimos la muestra con la que trabajamos valiéndonos de los cuatro ámbitos de estudio ya citados. 


\section{Resultados}

\subsection{Nivel académico y cualificación profesional}

\section{a.- Al ingresar en prisión}

Afirman saber leer y escribir la gran mayoría de los sujetos, aunque algo más del $9 \%$ se declara analfabeto.

En cuanto al nivel académico al ingresar en prisión, el 67\% de los internos declara no estar en posesión del Título de Secundaria Obligatoria. Ha ingresado con ese título el 26\% de los internos. Con Formación Profesional, en cualquiera de sus especialidades, ha ingresado el 5\% de los internos, y con Bachillerato ha ingresado sólo el $2 \%$ de los internos.

Figura 2.- Distribución del nivel académico al ingresar en prisión

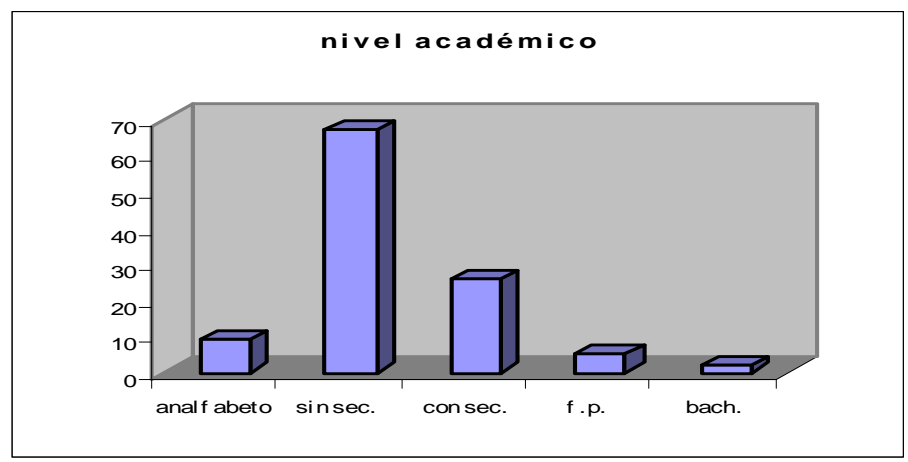

\section{b.- La mejora del nivel académico en prisión}

Es interesante conocer si existen internos que han aprovechado su tiempo en prisión para mejorar su nivel escolar y formativo por lo que hemos introducido una variable que nos permita conocer esta posible evolución.

Podemos observar que en lo referente a Formación Profesional y a Bachiller no se producen cambios. Sin embargo se produce una evolución de las personas que no tienen la titulación de Secundaria Obligatoria hacia los que tienen sí la tienen, pasando los primeros de ser un grupo de 138 personas a 132, y los segundos de 54 a 60. Por lo tanto, podemos decir que 6 internos de entre 206, han aprovechado su tiempo en prisión con una tarea educativa provechosa, la obtención del título de graduado escolar. Es 
decir, sólo el 2,9\% de los internos ha mejorado su nivel escolar. Esta cifra puede revelar el escaso interés que lo educativo despierta entre los internados, y es tarea de todos cambiar esta percepción e invertir esfuerzos en tareas educativas, porque pensamos que todo esfuerzo educativo, más tarde o más temprano dará sus frutos. En cualquier caso, aunque sólo el 2,9\% aprovecha su tiempo en mejorar su nivel educativo, no debemos despreciar esta evolución, que dice mucho de los internos a los que afecta.

\section{c.- Recuerdo de la infancia escolar}

Destacamos que 134 internos, el 65\% tiene un recuerdo bueno o muy bueno de su paso por la escuela, mientras sólo 16 internos, el 7,8\% tiene un recuerdo malo o muy malo de su pasado escolar. Se mantienen en una posición neutra, reconociendo que su paso por la escuela puede ser calificado como regular 56 internos, lo que supone el $27,2 \%$. Por lo tanto, podemos desprender de estos datos que la mayoría de los internos tienen un buen recuerdo de su paso por la escuela, a pesar del evidente poco aprovechamiento del tiempo escolar. Fueron bien tratados en ella y valoran su importancia, aunque no fueron capaces, en su gran mayoría, de seguir los ritmos de aprendizaje impartidos en ella, llevándoles a optar por el abandono escolar o por la no promoción de curso.

\section{d.- La profesión de los internos}

En cuanto a la profesión de los internos, ésta se distribuye del siguiente modo:

En las modalidades de albañilería, pintura, encofrados, y en definitiva todo lo relacionado con la construcción, se encuentra el 37,9\% de la población. Se declaran vendedores, preferentemente vendedores ambulantes el 18,9\%. A la especialidad de hostelería, principalmente como camareros o ayudantes de cocina, se dedica el $12,1 \%$. Ha elegido la pesca como profesión el 8,3\%. Se declaran sin profesión, y dicen que nunca han trabajado el $18,9 \%$. Existen algunos internos que dicen trabajar en otras ocupaciones, tales como mecánicos, en tareas de campo o como transportistas. 
Figura 3.- Distribución de la profesión de los internos

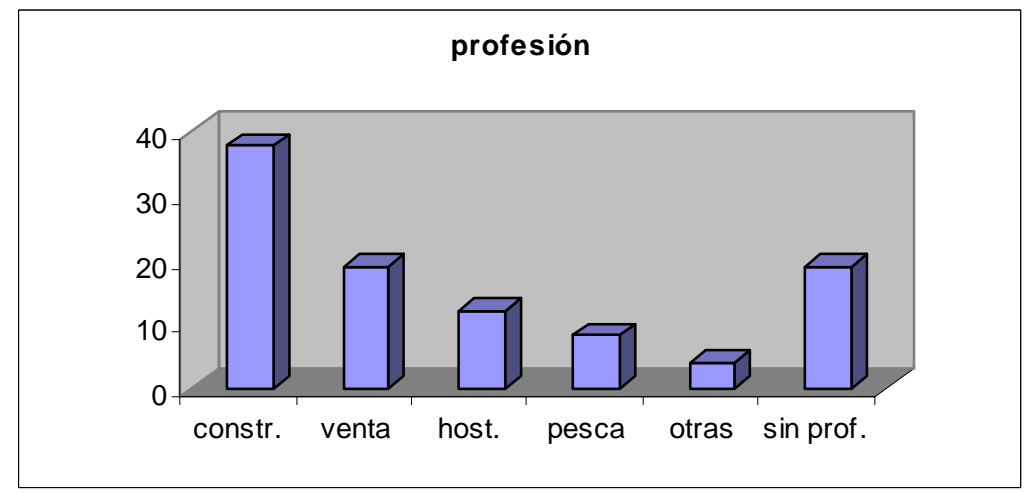

\subsection{Relaciones familiares}

En cuanto al estado civil de los internos, los resultados nos indican que existen 128 internos solteros, $(62,1 \%) ; 17$ casados, $(8,3 \%) ; 2$ viudos, $(1 \%) ; 7$ separados, $(3,4 \%)$ y 51 internos que afirman que conviven con una pareja, $(24,8 \%)$.

\section{a.- Antecedentes familiares en prisión}

El 56,3\% de los sujetos, afirma no tener antecedentes familiares en prisión, ya sean padres o hermanos, mientras que el $43,7 \%$ reconoce que sus padres o hermanos han estado ingresados en prisión.

\section{b.- Trato recibido de la familia}

Sólo siete internos, el 3,4\% reconoce haber recibido maltrato por parte de su familia, mientras que un número equivalente reconoce haber recibido un trato regular. Por último indicamos que 192 internos, el 93,2\% afirma haber recibido un buen trato por parte de su familia.

Si reconocemos como ciertas estas afirmaciones, podemos observar que además del maltrato recibido, causa que, sin duda, puede explicar la trayectoria delincuencial de los internos, también existen otras causas que conducen al delito. 


\section{c.- Trato dado a la familia y apoyo familiar}

En cuanto al trato que los internos han dispensado a sus familias, observamos que el $72,8 \%$, afirma haber tratado bien a sus familias.

Del total de sujetos, el 90,8\% afirma sentirse apoyado por sus familias, mientras que sólo el 7,3\%, dice no sentirse apoyado.

Creemos que recibir apoyo familiar en situaciones de encierro puede mejorar la forma de vida en las prisiones, ya que una forma de vida emocionalmente positiva, elimina tensiones y frustraciones, y por lo tanto puede favorecer actitudes positivas hacia los procesos de reeducación.

\section{d.- Nivel académico de los padres}

El nivel de estudios de los padres es una variable importante a tener en cuenta, ya que el ambiente de estudios en la familia puede influir en la valoración que los internos den a los mismos. Así, tenemos que, el 66\% de los padres y el 58,3\% de las madres, no han obtenido el Graduado Escolar. Sólo cinco padres, el 2,4\% y tres madres, el 1,5\% ha alcanzado la universidad. El resto se distribuyen entre padres "con Graduado Escolar", con "Formación Profesional" y con "Bachillerato".

\section{e.- Profesión de los padres}

En cuanto a la profesión de los padres, podemos observar que las profesiones a las que se dedican mayoritariamente los padres de los internos son la construcción, el $24,3 \%$, el comercio el $18,4 \%$, la hostelería, el 9,2\% y confiesan estar desempleados el $7,8 \%$.

En lo que se refiere a las madres, podemos destacar que el $45,1 \%$ son amas de casa, el 21,4\% se dedica a la limpieza, mientras que están desempleadas el 7,8\%. El resto se distribuye entre diferentes profesiones. Observamos que las profesiones de los padres son semejantes a las profesiones de los hijos, careciendo ambas de una elevada preparación y cualificación. 
Fig. 4.- Profesión de los padres

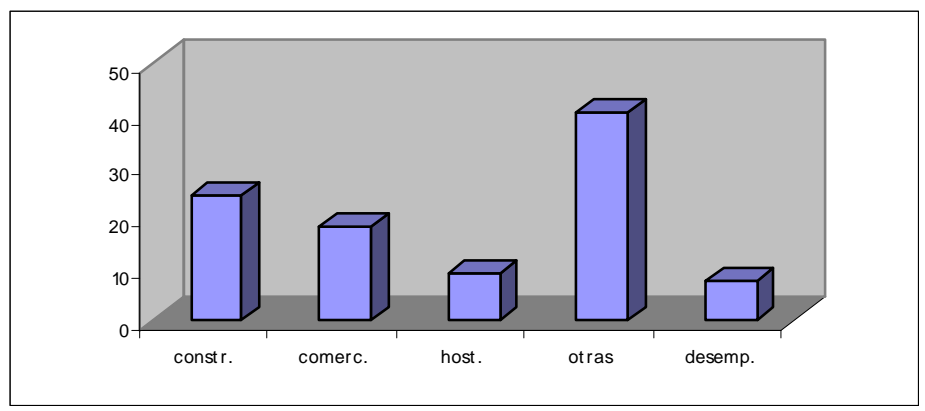

\subsection{Contactos con la marginalidad}

\section{a.- Consumo de drogas antes de ingresar en prisión}

Podemos concluir de los datos obtenidos, que el 88,8\% de los internos consumía drogas antes de ingresar en prisión, mientras que sólo el 11,2\% dice que no consumía. A partir de estos datos podemos aventurarnos a afirmar que los nuevos ingresos que se produzcan en prisión serán consumidores de drogas en un alto porcentaje, por lo que volvemos a insistir en la importancia del trabajo educativo preventivo que evite estas situaciones.

En cuanto al tipo de drogas consumidas, observamos que la gran mayoría de los internos confiesan un consumo politoxicómano, consumiendo asiduamente alcohol, hachís, cocaína, heroína y pastillas.

En cualquier caso, no sabemos si el consumo de drogas origina el acto delictivo y la entrada en prisión, pero sí sabemos que un gran porcentaje de los que ingresan en prisión consumen drogas con anterioridad a su ingreso. Si a esa circunstancia le añadimos otras que conforman el perfil de la muestra estudiada, podemos presuponer que el riesgo de entrar en la espiral del delito va aumentando progresivamente. 


\section{b.- Consumo de drogas en prisión}

En cuanto al consumo actual de drogas, dentro de los centros penitenciarios, nos encontramos que aproximadamente el $60 \%$ de los sujetos afirma consumir drogas en prisión.

\section{c.- Participación en programas de desintoxicación}

Respecto a la participación en programas de desintoxicación, ya sea en libertad o tras su ingreso en prisión, observamos que el 78,6\% nunca ha participado en estos programas, mientras que el $21,4 \%$ dice haber participado en algún programa. La Institución Penitenciaria prevé que los internos que así lo deseen, así como los que por su tratamiento, se prescriba, participen en estos programas de desintoxicación.

\section{Figura 5.- Distribución de la participación en programas de desintoxicación}

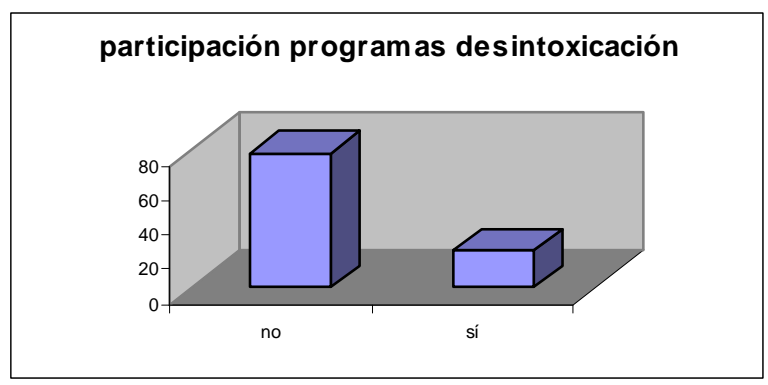

\section{d.- Ingresos en Centros de Menores}

Entre todos los sujetos, 58,3\% afirma que no han estado ingresados en Centros de Menores, mientras que el 41,7\%, reconoce haber estado alguna vez internado en estos centros.

Esta variable hay que relacionarla con la de primario/reincidente que veremos más adelante, y con la argumentación que allí se defiende. Así, los internos que han estado ingresados en centros de menores suelen tener una importante carrera delictiva, aunque por criterios cronológicos no hayan ingresado anteriormente en prisión.

En estos centros las personas comienzan a interiorizar pautas educativas y resocializadoras, que pueden impedir el posterior ingreso en prisión, o por el contrario, 
en estos centros comienzan un proceso de institucionalización que les acompaña durante gran parte de sus vidas.

\section{e.- Tipología delictiva}

Consideramos importante analizar el tipo de delito que mantiene a estos jóvenes en prisión, porque va a indicar la tipología delictiva de los internados, y realizando un estudio pormenorizado podemos llegar a saber si la tipología delictiva influye en la actitud mantenida por los internos ante los procesos reeducadores.

Así, tenemos que el 24,3\% se encuentra en prisión por la comisión de delitos contra la salud pública, relacionados con drogas. Se encuentra en prisión por delitos contra la propiedad, robos, el 56,3\%, mientras que el 19,4\% lo está por delitos contra las personas, agresiones.

Figura 6.- Distribución de la tipología delictiva

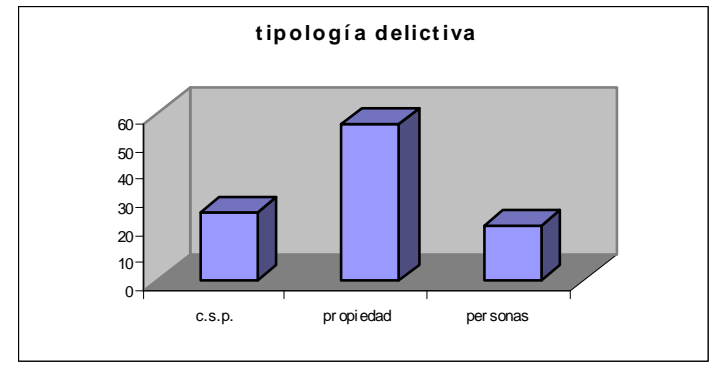

\section{f.- Uso de la violencia en el acto delictivo}

Resulta interesante saber si los internados en prisión han utilizado violencia a la hora de cometer el delito, ya que la violencia con que se desenvuelve una persona puede influir en la actitud mantenida por esa persona.

Así, tenemos que el 53,4\% dice no haber usado violencia al cometer el delito que lo mantiene en prisión, mientras que el 46,6\% reconoce haber usado violencia al cometer el delito. 


\subsection{Vida en prisión}

La población penitenciaria menor de veintiún años, según la actual legislación, debe permanecer en módulos separados del resto de la población reclusa adulta. Estos módulos de jóvenes deben tener una especial atención en los campos formativos, educativos, asistenciales y laborales. En definitiva, son módulos de especial atención e intervención. Los internos menores de veintiún años sólo deben realizar su vida fuera de estos módulos, si así lo propone motivadamente la Junta de Tratamiento y lo aprueba el Juez de Vigilancia

Penitenciaria. Por lo tanto, el paso de un módulo de jóvenes a un módulo de adultos debe ser la excepción en el Tratamiento Penitenciario. Sin embargo, podemos apreciar en los datos obtenidos, que 105 internos realizan su vida en módulos de adultos, esto es, el 51\% del total. Mientras que 101 internos, el 49\% lo hace específicamente en módulos de jóvenes.

\section{a.- Situación penal}

Del total de internos, el 64,1\%, está en situación preventiva, esto es, a la espera de la celebración del juicio que los condene a cumplir prisión o que los devuelva a la vida en libertad.

En situación de penados, es decir condenados, se encuentra el 35,9\% de los sujetos.

Debemos señalar que a los internos que se encuentran en una situación mixta, es decir, que tienen alguna causa penada, pero que también tienen una o más causas en situación preventiva, les indicamos que señalaran la opción de "penado", como su situación actual, ya que aun saliendo en libertad de los juicios que tengan pendientes, quedarían retenidos en prisión por la causa o causas penadas.

\section{b.- Situación penitenciaria}

En cuanto a la situación penitenciaria, del total de internos, han señalado la opción de "primario", es decir, señalan que es la primera vez que se encuentran en prisión el $64,1 \%$, mientras que señalan la opción "reincidente", es decir, internos que 
han estado más de una vez en prisión, el 35,9\%. Estas cifras coinciden con los de la variable "situación penal", aunque no tienen nada que ver.

\section{c.- Tiempo de estancia en prisión}

Sin lugar a dudas es muy importante para el tema que estudiamos, el tiempo de estancia de los internos en prisión, no sólo por los procesos reeducadores que la institución penitenciaria realiza o puede realizar con los internos, sino por el grado de deterioro que pueden sufrir los internos y que puede influir en la actitud mantenida por estos. Así, tenemos que del total de sujetos, el 77,2\% afirman llevar menos de un año en prisión, mientras que el 22,8\%, dicen llevar más de un año.

Estos datos nos indican que la mayoría de los internos no deben estar demasiado deteriorados por su situación y pueden ver el futuro de forma esperanzadora.

\section{d.- Actividad laboral en prisión}

En cuanto a la actividad laboral de estos internos en prisión, podemos destacar que aproximadamente el $50 \%$ no realiza ningún trabajo en prisión. Mientras que cerca del $40 \%$ limita su actividad laboral a tareas funcionales, es decir, su trabajo consiste en limpiar las zonas comunes y ayudar en aquellas tareas que se les solicita. Así, tenemos que sólo el $10 \%$ de los internos realizan algún trabajo productivo, remunerado y con las condiciones exigidas por la Seguridad Social.

\section{e.- Sentirse valorado}

Por otro lado, observamos que casi el 70\% de los jóvenes internados no se siente valorado ni recompensado por la actividad que realiza en los centros penitenciarios, siendo significativo el hecho de que más de la mitad no se sienten valorados por ellos mismos ni por sus parejas. Afirman que sus familias paternas valoran su actividad en torno al $45 \%$ de los casos, mientras que los compañeros de internamiento no valoran en nada su actividad en el $85 \%$ de los casos. Pero lo realmente preocupante es que consideran en casi un $30 \%$ de los casos que los educadores que trabajan con ellos no valoran nada su actividad. 


\section{f.- Responsabilidad en la comisión del acto delictivo}

Por otro lado, tenemos que el $61,2 \%$ de los internos, reconoce que ellos son los responsables de los delitos que han cometido y asumen su culpabilidad, haciendo copartícipe de esta responsabilidad en porcentajes similares al barrio, a los amigos y a personas con las que se relacionaban en la calle. Más del 90\%, dice que ni la escuela ni las relaciones familiares tuvieron que ver con su posterior caída en la delincuencia.

\section{g.- Motivaciones para delinquir}

En cuanto a las motivaciones que llevaron a los internos a cometer su primer delito, y por lo tanto a tomar el primer contacto con el mundo de la delincuencia y a acabar en prisión, tenemos que más del $50 \%$ dice haber cometido el primer delito alentado por el grupo de amigos, no existiendo necesidad económica ni necesidad de comprar drogas, aunque el fruto de los delitos se emplee en la compra de drogas o en adquirir productos que la sociedad consumista se empeña en hacernos ver como imprescindibles, ropas de marca, vehículos o cualquier otro producto superfluo que interiorizamos como indispensable. Para tener acceso a estos productos necesitamos dinero, y algunos jóvenes no dudan en apropiarse de lo ajeno para cubrir esta superflua necesidad.

\section{h.- Ocupación del tiempo libre en prisión}

Debemos señalar la importancia de la ocupación del tiempo libre como ocupación correcta del tiempo de ocio para que se produzca un verdadero proceso reeducador.

De los datos obtenidos podemos destacar que los mayores porcentajes se dan en la ocupación del tiempo libre a través de charlar, oír música, ver televisión, pasear y practicar deporte. 
También podemos destacar que la opción “leer”, como actividad preferida por los internados sólo ha sido escogida en 26 casos, es decir, sólo algo más del 12\% de la población pasa parte de su tiempo libre leyendo. Desde luego si pretendemos que la prisión sea un centro de educación o reeducación y de formación, la ocupación de apenas este $12 \%$ de la población en leer, no es lo más alentador, ya que la lectura se nos antoja fundamental en los procesos de formación y educación.

\section{Discusión y conclusiones}

En cuanto al nivel académico y cualificación profesional, es de destacar el bajo nivel académico de la población estudiada, por lo que parece claro que el bajo nivel académico y los acontecimientos delincuenciales, en muchos casos, están relacionados (cfr. San Juan, Ocáriz y de la Cuesta, 2.007) por lo que las intervenciones pedagógicas se antojan indispensables, o como medidas preventivas, o como medidas reeducativas, una vez ingresados en prisión. Además, una persona con una base cultural mínima, ofrece siempre la posibilidad de un tratamiento pedagógico provechoso, ya que está iniciado en el largo proceso de hacerse persona integrada en la sociedad.

También destacamos el bajo índice de internos con Formación Profesional, sólo el 5\%. Indudablemente una adecuada formación profesional podría reorientar los pasos de estos jóvenes hacia salidas profesionales que les ayudarían a evitar la entrada en prisión.

En cualquier caso, sería materia para reflexionar la posibilidad de permutar las condenas de los jóvenes en prisión, por la obtención de objetivos educativos (cfr. Calatayud, 2.004), al menos para algunos tipos de delitos. Recordamos que la actual Ley Penal del Menor, que afecta a los menores de dieciocho años, sí permite esta posibilidad.

Sea como sea, podemos apreciar que el nivel académico de los internados en centros penitenciarios no resulta demasiado alentador.

No cabe duda que el tiempo escolar es fundamental en la vida de todas las personas, mucho más en las que acaban pasando parte de ella en prisión. Creemos necesario que desde la escuela se realicen más esfuerzos y no dar ningún caso por 
perdido y trabajar, en el plano intelectual, aunque quizás parte del alumnado no sea capaz de superar cierto nivel, y sobre todo, en los planos emocional, convivencial, relacional y social, de manera que los alumnos reciban suficientes estímulos y argumentos como para que sus vidas no tomen rumbos que los lleven a prisión. Todos estos niveles de la personalidad están relacionados con lazos de dependencia mutua, por lo que la mejora en cualquiera de ellos repercute en todos los demás.

Por supuesto que esto debe ser tarea de la escuela, pero no exclusivamente de ella, y creemos que deben implicarse en estos procesos formativos-relacionales-sociales todas las instituciones y colectivos, siendo necesaria la perspectiva de la educación social, a través de los educadores de calle y de orientadores que trabajen en la orientación, estímulo y ayuda a los padres y también en la guía de estos jóvenes hacia salidas sociales que los alejen de la marginación, el delito y en definitiva de la prisión.

Respecto a las profesiones realizadas, podemos apreciar que ninguno de los trabajos antes relacionados exige una gran cualificación. Además, podemos observar que el tiempo trabajado en las profesiones antes citadas es corto, ya que el 27,2\%, declara haber trabajado menos de seis meses; entre seis meses y un año dicen haber trabajado el 29,6\% y más de un año, indican que han trabajado el 24,8\%. Por lo tanto, podemos suponer que en la mayoría de los casos son trabajos esporádicos o de temporada, que en ningún caso les permite afrontar el futuro con seguridad.

Indudablemente estamos hablando de jóvenes menores de veintiún años, por lo que es normal que su trayectoria profesional sea muy corta. Sin embargo, como ya vimos anteriormente, una gran parte de ellos no han alcanzado los niveles de estudios básicos. Por lo que debemos plantearnos que si no han estudiado ni tampoco han trabajado, ¿en qué han empleado el tiempo? La mayoría de ellos en no hacer nada, en el peor sentido de la palabra, ya que normalmente han pasado la vida reunidos en grupos, dedicándose a realizar actividades ilegales.

En lo que se refiere a las relaciones familiares, las variables estudiadas nos indican algunos hechos importantes:

En primer lugar podemos observar el alto porcentaje de la muestra cuyas familias no han tenido relación con el mundo de la delincuencia, por lo que podemos afirmar que se está produciendo un proceso de extensión delincuencial hacia capas sociales que hasta estos momentos no han tenido contacto con la delincuencia, y que socialmente son normalizadas, porque las prisiones, no son ya lugares donde sólo son

Revista Española de Investigación Criminológica 
ingresados los marginados, sino que cualquier persona, independientemente del grupo social o económico al que pertenezca, puede ingresar. Más aun si nos referimos a una población joven, con hábitos sociales que en muchos casos, y llevados por modas y tópicos, puede presentar actuaciones que lindan lo marginal e incluso lo delincuencial.

Por otro lado, tenemos al grupo de internos que reconocen antecedentes familiares en prisión. Este grupo de internos, son los que tradicionalmente han ocupado los centros penitenciarios, que pertenecen a clases desfavorecidas o marginales. Estos internos suelen sentirse orgullosos de las "hazañas" de sus padres o hermanos en prisión, y este hecho les confiere cierto estatus entre el resto de los internados.

A este último grupo de personas se le debe prestar atención en el campo de la prevención, ya que, sin lugar a dudas, pertenecen a grupos de riesgo que van a nutrir las prisiones generación tras generación, si no se realiza un trabajo educativo y preventivo con ellos.

Asimismo, tampoco debemos olvidar al grupo de internos cuyas familias no han tenido nada que ver con el mundo de la delincuencia, ya que si estas personas están sufriendo la situación de prisión, no es porque tengan malos modelos familiares, aunque no podemos descartar abandonos emocionales o educativos, sino debido, entre otras causas, a los modelos imitados de sus amigos, del barrio, y a la pérdida o confusión de valores que en el actual sistema social se producen, y que de un modo u otro, todos alentamos y reproducimos. Sin lugar a dudas, debemos trabajar en nuestras escuelas una educación en valores que ayude y oriente a los jóvenes a elegir opciones positivas de vida.

En lo relativo al nivel de estudios de padres y madres, podemos apreciar que en las familias de los internos no se ha dado demasiada importancia a los estudios. Esto, sin duda, influye en la valoración que hacen los internos de los estudios y de la escuela. Y aunque el nivel de estudios de los padres, no garantiza una vida al margen del delito, es seguro que junto a otras variables, ofrece a las personas la posibilidad de un hogar sano en todos los sentidos.

Respecto al estado civil, es bastante significativo observar que si sumamos los internos casados con los que dicen convivir con su pareja, obtenemos porcentajes superiores al 30\% de personas que dicen tener una familia propia. Hay que recordar que hablamos de jóvenes de 18, 19 ó 20 años y que además están en prisión, por lo que sus parejas, cuyas edades serán aproximadamente las mismas y sus posibles hijos se deben

Revista Española de Investigación Criminológica

Artículo 3, Número 6 (2008) $\quad$ www.criminología.net

ISSN: $1696-9219$ 
encontrar en unas situaciones no demasiado halagüeñas. Sin lugar a dudas, estas situaciones generan unas responsabilidades difíciles de sobrellevar. Creemos que la educación, en la familia y desde la escuela, deben realizar una función de información y orientación que evite estas situaciones, porque que sepamos existe un buen número de cónyuges de internos que están en la calle, sin el apoyo de su pareja, sin contar con el posible número de hijos cuya única relación con su padre o madre se produce a través de un cristal y una vez por semana. Evidentemente no es esta la mejor forma de educar a unos hijos. Además, esta situación suele tener como consecuencia que padres, hijos, tíos, sobrinos, nietos y abuelos, más veces de las deseadas, convivan en prisión.

En lo que concierne al contacto con la marginalidad, el porcentaje de consumidores de drogas en prisión denota una evolución positiva de los internos, aunque debemos tener presente que los datos obtenidos en esta variable quizás se encuentran sesgados, ya que los internos pueden pensar que si se declaran consumidores de drogas dentro de los centros penitenciarios pueden ser perjudicados en su evolución y que su libertad puede retrasarse, por lo que quizás el porcentaje sea más alto. En cualquier caso, el consumo de drogas puede influir decisivamente en las actitudes mantenidas hacia los procesos de reeducación en prisión.

En relación al tipo de drogas consumidas dentro de los centros penitenciarios, destacamos que los mayores consumos se detectan en hachís y pastillas. Percibimos una reducción de los consumos de heroína, cocaína y alcohol a menos del 10\% de la población con la que trabajamos. Estos porcentajes tan bajos, más que a la renuncia de los internados a consumirlas, puede deberse a la dificultad de introducir estas sustancias en el interior de los centros penitenciarios.

Indudablemente, nos parece que la participación en programas de desintoxicación es importantísima para que se produzcan procesos reeducadores y de reinserción social, ya que estos programas además de conseguir disminuciones en los consumos, aportan estabilidad emocional, aumentan la autoestima y evitan deterioros, y todos estos procesos son indispensables para la consecución de la reinserción social, por lo que la ausencia de estos programas en la vida de los internos dificulta las tareas reeducativas.

Por otro lado, debemos considerar que quizás el concepto que tienen los internos de los actos violentos no es el mismo que el que podemos tener las personas 
socialmente normalizadas. Esta afirmación se desprende de conversaciones mantenidas con internos que afirman que ciertos actos que podemos considerar violentos, ellos lo definen como "defensa de lo que les pertenece, o de lo que quieren obtener", reduciéndose todo a la supervivencia, sea como sea, dentro del grupo.

En cuanto a la vida en prisión, observamos que a menudo los internos que salen de los módulos de jóvenes hacia módulos de adultos, lo hacen por uno de estos dos motivos:

- Son internos considerados muy buenos, que reciben influencias perniciosas y malos tratos por parte de los demás internos del módulo de jóvenes, y a los que la dinámica de este módulo no hace más que perjudicarles.

- Son internos mal adaptados al módulo y a la vida dentro de éste, que interfieren negativamente en el buen desarrollo de la vida del módulo, perjudicando al resto de los internos.

En cualquiera de los dos casos anteriores, la Junta de Tratamiento propone y el Juez de Vigilancia Penitenciaria aprueba la separación del joven del resto de los internos del módulo que ocupaba para ubicarlo en un módulo de adultos.

Sea como sea, creemos que los datos obtenidos señalan un porcentaje demasiado alto de internos menores de veintiún años que viven fuera de los módulos en los que tienen que realizar su vida según la vigente legislación, ya que es en este tipo de módulos donde debe existir una mayor actividad y atención educativa hacia los internos.

Asimismo, debemos considerar que existen internos primarios, que efectivamente son nuevos en el mundo delincuencial. Otros, sin embargo, tienen una larga trayectoria de delitos, no habiéndose producido su ingreso en prisión anteriormente, por motivos cronológicos, ya que como señalamos anteriormente, con la entrada en vigor de la Ley Penal del Menor en el año 2.000, los menores de 18 años no ingresan en prisión, sino que dependiendo de los hechos por los que se les condene son ingresados en Centros de Menores o se les permuta la pena por medidas socioeducativas, pero en cualquier caso, la gran mayoría no son recién llegados al mundo de la delincuencia.

La reincidencia y la multireincidencia son, sin lugar a dudas, variables a tener muy en cuenta a la hora de analizar los procesos de reeducación de los jóvenes 
internados en prisión, así como la forma de percibir la reeducación y la reinserción social.

También es importante comprobar el tiempo que los internados en prisión mantienen entre ingresos, es decir, los tiempos de libertad entre ingresos. Así tenemos, que el período donde más casos se dan, están entre los tres y los siete meses. Por lo tanto, la mayoría de internos que tienen más de un ingreso en prisión, pasa en libertad entre los ingresos, períodos que oscilan entre los tres y los siete meses.

En cuanto a las actividades que realizan en prisión, debemos tener en cuenta que, los porcentajes antes citados, se refieren a la realización de una sola actividad, por ejemplo, la de ir a la escuela, como de varias, es decir, existen internos que compaginan varias actividades, por ejemplo, ir a la escuela, asistir a cursos formativos y practicar actividades deportivas.

En lo referente a la actividad laboral en prisión, creemos, que si queremos potenciar la reeducación y la reinserción social de los jóvenes internados en prisión debemos potenciar las tareas laborales de los mismos, ya que no es posible concebir la reinserción sin ocupación laboral, por lo que creemos que los datos obtenidos reflejan que el sistema penitenciario actual no se orienta hacia esa pretendida reeducación y reinserción social, porque ¿cómo vamos a reinsertar si sólo realizan en prisión una actividad laboral productiva un $10 \%$ de la población?

En cuanto a la variable sentirse valorado, podemos concluir que los internos que realizan actividades en prisión no se sienten, en su mayoría, valorados ni recompensados, ni por ellos mismos ni por los demás internos, y lo que es peor, en un gran número de casos tampoco se sienten valorados por los educadores que trabajan con ellos. Con estos datos, observamos la evidencia de que no existe una buena comunicación ni una buena transmisión de sensaciones entre los trabajadores de la institución penitenciaria y los internados en prisión. Asimismo, creemos que puede existir una mala percepción de las emociones de los educadores penitenciarios por parte de los internos. En cualquier caso, no sentirse valorados dificulta las tareas educativas y de reinserción social.

Debemos destacar que más del 50\% de los internados dicen que la sociedad nada tuvo que ver con que llegaran a delinquir. Quizás este punto necesite un análisis más profundo, porque, como ya vimos, una gran cantidad de internos está en prisión por delitos contra la propiedad, robos. Haciendo un ejercicio de reflexión y de conciencia,

Revista Española de Investigación Criminológica 
¿por qué no relacionar estos robos con el bombardeo consumista al que la sociedad nos somete?, ya que suele existir una gran desproporción entre el estímulo consumista y las oportunidades de obtener un nivel de ingresos que permita consumir. Pero también hay que tener presente que las actividades delictivas se extienden por todas las clases sociales, por lo que quizás más peso que el propio consumo, tenga la confusión existente en nuestra sociedad en lo que se refiere a los valores. Quizás, aunque no sea reconocido fácilmente, la sociedad, su estructura, valores y funcionamiento sí tengan algo que ver con los resultados delincuenciales actuales, por lo que debemos trabajar en nuestro entorno, con nuestra forma de vida y con la prelación de nuestros valores para mejorar la cuota parte de la sociedad que nos toca.

Ante estos datos podemos concluir, y según las percepciones de los internos, que se presentan como agentes motivadores que les han llevado a cometer delitos, el mismo interno, los amigos, el barrio, y en alguna medida la actual sociedad.

De este modo, se nos presentan tres campos de trabajo en tareas preventivas, que en definitiva se reducen al ámbito de los barrios más o menos marginales, ya que si trabajamos preventiva y educativamente en estos barrios, trabajamos con los individuos y con sus grupos de iguales.

En cualquier caso, podemos deducir que la motivación principal para cometer el primer delito la encontraron en sus amigos, por lo que volvemos a insistir en la importancia que se debe dar al trabajo preventivo a través de la educación social, con los grupos de jóvenes en los barrios.

Asimismo, debemos cuestionarnos la viabilidad de los centros penitenciarios como lugares de reeducación, porque parece evidente que para reeducar, educar, en definitiva, es necesario un ambiente de libertad, y desde el encierro de un centro penitenciario parece improbable acometer procesos educativos. Del mismo modo, parece improbable acometer procesos de reinserción social, inserción social, inclusión, separando con un muro, segregando a la población a la que pretendemos reinsertar, ya que para reinsertar, insertar, necesitamos del contacto de la sociedad con las personas aisladas, y estos procesos desde un centro penitenciario parecen de difícil ejecución.

Creemos, sin embargo, en el componente pedagógico y educativo que debe presidir las actuaciones en los centros penitenciarios, y que deben llevar aparejada la consecución de la normalización de la conducta de los internados, a través de los valores morales y éticos de una sociedad democrática.

Revista Española de Investigación Criminológica

Artículo 3, Número 6 (2008) $\quad$ www.criminología.net

ISSN: $1696-9219$ 
Así, y aún considerando simplista la explicación de que los problemas de marginación, exclusión social, inadaptación y delincuencia, son únicamente debidos a déficits educativos, pensamos que desde la pedagogía social y con un enfoque educativo, sí se pueden aportar soluciones que palien estos estados carenciales.

Parece necesaria la intervención desde la pedagogía social, y desde este ámbito debemos estudiar y diseñar intervenciones que favorezcan formas de convivencia más justas, solidarias y libres, y menos violentas, excluyentes e insolidarias, no por mera protección de los jóvenes, sino por motivos de justicia social (Martín, 2006).

No cabe duda que estos planteamientos quedan lejos de las condiciones de vida de un centro penitenciario, pero no por ello debemos renunciar a su implantación, porque el discurso pedagógico tiene que ser, por naturaleza, potencialmente utópico, ya que, como nos indica el profesor Sáez (2.003), esto nos permite cargar de valores e ideales nuestras intervenciones.

Optamos por la conversión de la Institución Penitenciaria en una institución educativa donde se implante un modelo pedagógico con una intervención más humana.

Consideramos que el ingreso en prisión de personas, es el último argumento al que debemos recurrir para resolver nuestros problemas sociales, ya que como nos indica Javier Urra (1.997), con el ingreso en prisión se evita el mal, pero no se proporciona el bien.

Creemos que debemos intervenir con actuaciones pedagógicas encaminadas a la prevención de la reincidencia, respetando escrupulosamente los derechos humanos de los internados, a través de una educación en la ciudadanía y en los valores democráticos, con intervenciones pedagógicas que nos conduzcan hacia la reeducación. 


\section{Referencias}

Amorós, P. y Ayerbe, P. (Eds.) (2.000): Intervención educativa en inadaptación social. Madrid: Síntesis.

Beristain, A. (1.985): Ciencia penal y criminología. Madrid: Tecnos.

Bolívar, A. (1.995): La evaluación de valores y actitudes. Madrid: Anaya.

Calatayud, E. (2.004): Agravar las penas no disminuye la delincuencia. Conferencia pronunciada en la Universidad de Alicante.

Constitución Española de 31 de octubre de 1978. (B.O.E. nº 311, de 29 de diciembre).

Escámez, J. y Ortega, P. (1.986): La enseñanza de actitudes y valores. Valencia: Nau Llibres.

Foucault, M. (1.976): Vigilar y castigar. México: Siglo XXI.

García, J. y Sancha, V. (1.986): Psicología penitenciaria. Áreas de intervención terapéutica. Madrid: UNED.

Hair, J; Anderson, R.; Tatham, R. y Black, W. (1.999): Análisis multivariante. Madrid: Prentice Hall Iberia.

Kaiser, G. (1.988): Introducción a la criminología. Madrid: Dykinson.

Kleinbaum, D.G. (1.994): Logistic Regresion. New York: Springer Verlag.

Ley Orgánica 5/2.000 de 12 de enero, reguladora de la responsabilidad penal de los menores.

Martín V.M. (2.006): Actitudes de los internados en prisión, menores de veintiún años, ante la función reeducadora del medio penitenciario en el ámbito andaluz. Málaga: Servicio de Publicaciones de la Universidad de Málaga.

Martínez, R. (1.999): El análisis multivariante en la investigación científica. Salamanca: Hespérides.

Pinillos, J.L. (1.990): Cuestionario de personalidad CEP. Madrid: TEA ediciones.

Sáez, J. (2.003): La profesionalización de los educadores sociales. En busca de la competencia educativa cualificadora. Madrid: Dykinson.

San Juan, C.; Ocáriz, E. y de la Cuesta, J.L. (2.007): Evaluación de medidas en medio abierto del Plan de Justicia Juvenil de la Comunidad Autónoma del País Vasco, en Boletín Criminológico, $n^{o}$. 96. Instituto Andaluz de Criminología.

Silva, L.C. y Barroso, I.Ma . (2.004): Regresión Logística. Cuadernos de Estadística, $n^{o}$. 27. Salamanca: Hespérides.

Urra, J. (1.997): Violencia. Memoria amarga. Madrid: Siglo XXI.

Revista Española de Investigación Criminológica

Artículo 3, Número 6 (2008) $\quad$ www.criminología.net

ISSN: $1696-9219$ 


\section{Currículo del autor}

Víctor Manuel Martín Solbes es Doctor en Pedagogía por la Universidad de Málaga. Compatibiliza su labor como Educador Social de Instituciones Penitenciarias con la de Profesor Asociado en el Departamento de Teoría e Historia de la Educación de la Facultad de Ciencias de la Educación de la Universidad de Málaga, donde ejerce docencia en las titulaciones de Magisterio, Educación Social, Psicopedagogía y Pedagogía. Realiza investigaciones relacionadas con el mundo penitenciario, las drogodependencias, la exclusión social y la interculturalidad, desde una perspectiva sociopedagógica. 